\title{
La digitalización de la imagen cinematográfica y la ausencia de estándares tecnológicos
}

\author{
Oswaldo GArcía CRESPO \\ Universidad de Vigo \\ oswaldogarciacrespo@gmail.com
}

Recibido: $24 / 07 / 2012$

Aceptado: 25/10/2012

\section{Resumen}

Este artículo aborda la digitalización de los procesos productivos cinematográficos para analizar uno de sus principales retos, el establecimiento de estándares digitales para le tratamiento de la imagen. Con ello se establece una relación entre tecnología digital y la evolución del cine como actividad empresarial en un entorno altamente competitivo.

Palabras clave: Digitalización, estándares, procesos productivos, optimización.

\section{Digitalization of film image and the lack of technological standards}

\begin{abstract}
The purpose of this study is to analyze the digitalization of film production processes focusing on one of its major problems, the establishment of digital standards on the realm of image manipulation. This paper aims at relating digital technology and the development of cinema as a business activity in a highly competitive enviroment.
\end{abstract}

Keywords: Digitalization, standards, production processes, optimization.

\section{Referencia normalizada}

GARCÍA CRESPO, Oswaldo (2012): "La digitalización de la imagen cinematográfica y la ausencia de estándares tecnológicos”. Estudios sobre el mensaje periodístico. Vol. 18, núm. especial octubre, págs.: 337-346. Madrid, Servicio de Publicaciones de la Universidad Complutense.

Sumario: 1. Introducción. 2. El dinamismo del objeto de estudio. 3. La ausencia de estándares digitales en el tratamiento de la imagen cinematográfica; 3.1. El formato; 3.2. La digitalización de la imagen cinematográfica; 3.2.1. Digital Intermediate y producción de efectos visuales; 3.2.2. La digitalización del rodaje; 3.3. Flujos de trabajo estandarizados y optimizados. 4. Conclusiones. 5. Referencias bibliográficas

\section{Introducción}

El factor tecnológico en el ámbito de los film studies, suele ganar presencia en épocas de grandes cambios. La introducción del cine sonoro, el color o en nuestro caso, la digitalización de la imagen cinematográfica, constituyen hitos que dan pie al desarrollo de trabajos académicos que, en la mayoría de los casos, ponen su foco en las consecuencias formales o estéticas derivadas de la implementación de una determinada tecnología. El presente trabajo, sin embargo, estudia los problemas derivados de la aplicación de tecnología digital sobre los procesos productivos. Para ello se analiza la implementación de la tecnología digital y su repercusión a través de uno de sus puntos débiles, la ausencia de estándares, que estaría provocando una falta de flexibilidad y control sobre los procesos productivos. Es por tanto un estudio más centrado en la naturaleza industrial del cine que en su vertiente expresiva, asumiendo la posibilidad de establecer una separación entre procesos productivos y las ideas y emociones que acompañan al lenguaje cinematográfico. 
El registro y manipulación de la imagen cinematográfica nunca se han limitado a un único formato. De hecho siempre han coexistido diferentes formas de acceso, manejo y reproducción. Y si bien la introducción de tecnología digital en el ámbito cinematográfico ha supuesto una mejora generalizada de los procesos productivos en términos de calidad y coste, ésta podría no presentar una influencia positiva en todos los casos. Existen ciertas desventajas que Swartz (2005: 4-5) sintetiza en cuatro factores directamente relacionados con la producción de cine digital:

- Una inmadurez que implica cambios y adelantos constantes, que si bien pudieran parecer positivos en un primer momento, implican la obsolescencia de productos y procesos.

- Los altos costes que conlleva una renovación constante del hardware.

- La complejidad de la imagen digital, incapaz de albergar márgenes de error en comparación con la analógica.

- La gran volatilidad de los formatos de grabación de imagen digital y la vulnerabilidad de los espacios físicos de almacenamiento.

Debido al alto componente tecnológico del presente trabajo y la volatilidad de la tecnología digital, consideramos fundamental la utilización de fuentes bibliográficas actualizadas y revistas especializadas del sector. Y si bien está selección resulta posible gracias en gran medida a publicaciones del ámbito anglosajón, la enorme velocidad con la que se están introduciendo herramientas digitales en le mercado invitaba al desarrollo de una estrategia metodológica complementaria a la revisión de fuentes documentales, capaz de dar respuesta a este dinamismo.

\section{El dinamismo del objeto de estudio}

La estrategia desarrollada para abordar nuestro objeto de estudio ha dado lugar a una estructuración de la investigación en dos fases. Cada una de estas fases han sido acometidas de forma secuencial, haciéndose patente la existencia de diferencias metodológicas entre ellas.

Una primera fase aborda el estudio de los principales formatos digitales de tratamiento de la imagen a través de fuentes bibliográficas especializadas en tecnología cinematográfica, la mayoría de ámbito anglosajón. Complementamos esta búsqueda con revistas especializadas y fuentes electrónicas dirigidas a profesionales del medio, cuyo principal objetivo es dar soporte y soluciones tecnológicas a flujos de trabajo asociados fundamentalmente a la fase de postproducción.

Una vez construida una base sólida de carácter tecnológico, se procede obtener un acercamiento mayor a la realidad industrial que unida a nuestra propia experiencia profesional, nos permita elaborar una visión realista del problema. Tras una aproximación a estudios similares, centrados en procesos productivos cinematográficos con un alto componente tecnológico, como en el Spelthann y Haunschild (2011) o el de Ciller (2009), se toma de decisión de configurar un panel de expertos para la realización de 
entrevistas ${ }^{1}$. Este acercamiento a la realidad industrial del cine se materializa en un trabajo interpretativo y exploratorio en el que las opiniones de los profesionales del sector ocupan un lugar protagonista. Así, se han planteado una serie de criterios que, combinados, pretenden conseguir alcanzar un alto grado de representatividad.

- Deben ser profesionales con una trayectoria lo suficientemente amplia como para haber asistido al proceso de digitalización de la fase de postproducción comenzado en España entre finales de la década de los 90 y principios de siglo XXI.

- Tendrán preferencia los perfiles profesionales que despiertan sinergias con los objetivos de esta investigación; aquellos que centran su trabajo en le tratamiento de la imagen digital y aquellos encargados de gestionar los procesos productivos.

- Se tendrá en consideración que hayan participado en películas premiadas por la Academia del Cine. Se valorará asimismo el éxito comercial y el que hayan recibido premios a su labor profesional.

- Se tendrán en cuenta la relevancia de las producciones en las que hayan participado en la medida en que éstas constituyan ejemplos paradigmáticos en cuanto a la digitalización de la imagen cinematográfica, tanto en la fase de postproducción como de rodaje.

Tanto los criterios de selección de los entrevistados como el cuestionario han sido desarrollados con la intención de extraer información que será destinada a la elaboración de este artículo, así como para el desarrollo de otros trabajos centrados en el uso de tecnología digital en la fase de postproducción cinematográfica. Por ello, en este artículo no aparecen todos los profesionales entrevistados, pues sólo las respuestas de algunos de ellos han despertado sinergias con el objeto de estudio que nos ocupa en este caso.

\section{La ausencia de estándares digitales en el tratamiento de la imagen cinemato- gráfica}

La investigación parte del estudio de los formatos para describir un contexto productivo en el que la imagen digital ejerce una influencia mayor sobre las rutinas de producción cinematográficas. Por ello se aborda la producción de efectos visuales, el Digital Intermediate y la digitalización del celuloide para la descripción de un escenario industrial que lejos de resolver la ausencia de estándares por sí solo, tiende a introducir cada vez más herramientas y nuevas especificaciones, generando incompatibilidades y debilitando el entramado tecnológico.

La elección del formato de imagen digital conlleva el establecimiento de un punto de partida que condicionará las rutinas de producción, ejerciendo a su vez una influencia directa sobre gestión de los recursos de los que disponen los proyectos cinematográficos.

1 Así, se elaboró un cuestionario que cumplía con los objetivos asociados a "la entrevista estructurada" como estrategia científica para investigaciones en el ámbito de la comunicación tal y como es concebida por De Miguel (2005: 252). 


\subsection{El formato}

En el transcurso de una producción cinematográfica, las decisiones relacionadas con el formato son un aspecto fundamental, de forma que en las productoras de postproducción no es extraño encontrar departamentos dedicados a la gestión de la información que conlleva la manipulación digital de la imagen.

"... is as much a matter of economics, marketing and aesthetics as it is a question of functionality. A format is thus the provisional crystallization of a complex set of histories, processes and interests". (Bennett et al., 2008: 19).

Es necesaria la elección del formato adecuado para cada producción en la medida en que va a determinar la cantidad de espacio de almacenamiento que requerirá el proyecto, el ancho de banda de la red de comunicación, la velocidad de bajada y los tiempos asignados a cada etapa (Wright, 2008: 34). Por tanto, la decisión acerca del formato debería ser tomada en la fase de preproducción por el director y el director de fotografía, además de poder estar involucrados el productor y el supervisor de efectos especiales ${ }^{2}$. Así, la elección del formato no sólo determinará cuestiones de carácter creativo, sino que condicionará el flujo de trabajo en postproducción y sus costes.

En este contexto, los sistemas de compresión utilizados van a condicionar en gran parte la toma de decisiones ${ }^{3}$. En un contexto productivo, cuando hablamos de compresión nos estaremos refiriendo a transportar la misma información en la menor cantidad de tiempo o con la mayor velocidad de datos posible tratando de: “... realizar algún proceso que sería imposible sin la compresión y realizar un proceso conocido de un modo más económico" (Fernández y Nohales, 2004: 76). La compresión hace que el flujo de información sea manejable. El tamaño de los archivos de imagen resulta por tanto fundamental en términos de optimización del flujo de trabajo, por ello cobra importancia el desarrollo de líneas de investigación y desarrollo dedicadas al diseño de sistemas que reduzcan el peso de los archivos, minimizando el impacto sobre su calidad.

La velocidad que otorgan al flujo de trabajo los sistemas de compresión no sólo es positiva en tanto en cuanto supone una reducción de tiempo y $\operatorname{costes}^{4}$, también facilita el proceso creativo, permitiendo probar ideas y efectos más elaborados. Es un aspecto técnico de gran importancia cuya gestión se está viendo limitada por no existir acuerdos entre fabricantes y desarrolladores que permitan adaptar las decisiones tomadas a entornos tecnológicos que garanticen la fiabilidad y compatibilidad en todo

2 Si bien Squires (2010: 465-466) defiende este planteamiento para la industria americana, no creemos que sus diferencias con respecto a otras industrias conlleve variaciones en este sentido.

3 En la mayoría de los contextos códec y formato pueden ser utilizados como sinónimos.

${ }^{4}$ Para Case (2010: 200) este hecho está condicionado por las presiones económicas de la industria, que pueden forzar a la elección de un formato digital de grabación poco pesado en aras de un abaratamiento de la película, por encima de cuestiones relativas a la calidad de la misma, que pasaría a un segundo plano. 
el pipeline ${ }^{5}$. La necesidad de establecer una estructura sólida a nivel tecnológico es urgente en la medida en que asistimos a una evolución en la que la imagen digital está aumentando su presencia tanto en la fase de producción como en la de postproducción.

\subsection{La digitalización de la imagen cinematográfica}

Los sistemas de captación de imagen digital están sustituyendo al celuloide de forma cada vez más rápida. Incluso en las producciones que utilizan el fotoquímico, la postproducción es digital ya en la gran mayoría de los casos, dando lugar a procesos englobados bajo el término Digital Intermediate, facilitando la generalización del uso de $V F X$ (efectos visuales digitales) y aumentando en consecuencia el volumen de información digital para la gestión de la imagen cinematográfica ${ }^{6}$.

\subsubsection{Digital Intermediate y producción de efectos visuales.}

El DI (Digital Intermediate) es un sistema de trabajo que parte de la digitalización de todo el negativo obtenido durante la grabación y no sólo del montaje, como se realizaba tradicionalmente. Se trata en esencia de una sustitución del material fotoquímico por archivos digitales. Las ventajas de su uso residen en el rápido acceso al material y una mayor seguridad en la gestión del mismo (James, 2006: 7-9). Desde el punto de vista global de la producción, su valor se centra en la simplificación en términos de tiempo y dinero, en un mayor control y seguridad sobre el negativo de la grabación y un mayor control creativo para la corrección de color (Wright, 2008: 223). Este último aspecto es para Brinkman (2010: 460) la característica más reseñable del DI: Permitir a los profesionales tener un mayor control sobre la imagen final poniendo a su disposición una gran variedad de herramientas para la gestión del color, contraste, nitidez y grano que en un contexto analógico sería inimaginable.

Sin embargo, su utilización implica un flujo de trabajo complejo a nivel técnico en el que todas las partes deben coordinarse, adaptándose a las necesidades específicas en términos de formato, estructuras de directorio y nomenclatura. Esta coordinación es especialmente intensa con el departamento de producción de VFX (efectos visuales). Este apartado de la producción cinematográfica, el de los efectos digitales, aumenta año a año, provocando una tendencia a trasladar la creación de la imagen cinematográfica de la fase de rodaje a la postproducción.

La utilización de fondos virtuales, la duplicación de figurantes y el borrado digital de elementos innecesarios en localizaciones naturales forman parte de estrategias de producción habituales. Estas soluciones de producción reciben el nombre de efectos visuales invisibles. Todas estas técnicas conllevan una manipulación de la información digital en diversas fases, haciendo aumentar la complejidad técnica de los

5 El término se refiere a las especificaciones técnicas sobre las que descansa un flujo de trabajo (Novy, 2010: 786).

${ }^{6}$ En la actualidad es ya habitual, en el caso de que se haya rodado en fotoquímico, la digitalización de todo el material debido a que los precios asociados a la digitalización son menores que los que derivados de la utilización de tecnología analógica en postproducción (M. Briozzo, entrevista personal, 22 de marzo, 2012). 
procesos necesarios para llevarlos a cabo. La generalización en el uso del $D I$ y de $V F X$ está adelantándose al establecimiento de un escenario tecnológicamente sólido, que permita el desarrollo de especificaciones técnicas globales y no el desarrollo de herramientas destinadas a solucionar una tarea concreta, sin tener en cuenta que debe ser implementada con éxito en una compleja cadena productiva.

\subsubsection{La digitalización del rodaje}

La reestructuración de Kodak es sintomática de un contexto en el que los sistemas digitales de captación representan la solución más eficiente en la gran mayoría de los casos. También está estandarizado el uso de cámaras $D S L R^{7}$ en producciones de guerrilla, es decir, películas a "coste cero" que ven en estas cámaras fotográficas, con prestaciones para la grabación de video en $\mathrm{HD}^{8}$, la posibilidad de alcanzar niveles de calidad que hace pocos años eran inasumibles ${ }^{9}$.

En el 2010, aproximadamente la mitad de los rodajes en España se realizaron con cámaras digitales ("La producción de largometrajes con medios digitales en España se estabilizó en 2010", 2011: 14) ${ }^{10}$. Aún a falta de datos actualizados del 2011, las revistas especializadas del sector se refieren a un abandono progresivo de los formatos analógicos de grabación en España. La mejora en la calidad de los formatos digitales se está produciendo de forma constante y en todas las fases, de modo que parece una cuestión de tiempo que todo el flujo de trabajo, desde la grabación hasta la proyección sea digital en la mayoría de los $\operatorname{casos}^{11}$. La industria cinematográfica española no parece que vaya a ser una excepción en este sentido ya que desde mayo del 2009 a abril del 2011, el número de salas digitales se cuadriplicó. Así, se estarían produciendo una revolución tecnológica compartimentada "producción-exhibición", provocando una búsqueda de la compatibilidad a posteriori, en lugar de establecer las bases tecnológicas sobre las que construir sistemas integrados y flexibles que garanticen la compatibilidad entre todas las partes y un modelo de crecimiento a medio plazo estable en lo tecnológico.

7 Cámaras réflex digitales. Del inglés Digital Single Lens Reflex.

8 Resolución 1080p en la Canon 5D Mark 2 y en la Nikon D800.

9 También es posible encontrar estas cámaras en grandes producciones como The Avengers (Whedon, 2012), si bien su uso responde a necesidades muy específicas, en las que la merma en la calidad de la imagen se justifica por cuestiones de carácter narrativo. En Witmer (2012: $35)$. Sin embargo en ocasiones su uso puede acarrear problemas en la proyección, como en El perfecto desconocido (Bestard, 2011). En este caso se utilizó una Canon 5D para grabar los flashbacks de uno de los personajes. Para Nicolás Pinzón, director de fotografía de la película, en estas partes se nota un cierto pixelado. En Larumbe (2012: 30-31).

${ }^{10}$ De las revistas especializadas consultadas, Cineinforme es la que trata de establecer más análisis cuantitativos en este sentido, si bien su fuente de información es el ICAA.

${ }^{11}$ Según un informe del IHS a través del Screen Digest, al que Cineinforme ha tenido acceso, en el año 2011 el total de salas de exhibición digitales ha superado por primera vez a las analógicas en el mundo. ("El 25 por ciento de las salas de España están digitalizadas", 2011, p. 34). 


\subsection{Flujos de trabajo estandarizados y optimizados}

La constante introducción de herramientas digitales en la producción cinematográfica unida a una ausencia de estándares que garanticen la compatibilidad entre todas las partes, impiden poner en marcha estrategias fiables de control sobre la solidez tecnológica de todo la producción ${ }^{12}$.

"Technology standards change continuously and even the momentary standard software packages are customized anew for each individual project; skills and competencies in production are thus rather transient in nature". (Spelthann y Haunschild, 2011: 104).

Ferrán Piquer (entrevista personal, 1 de febrero, 2012) alude a la enorme inseguridad que genera la falta de estándares de almacenamiento y seguridad del material de grabación ${ }^{13}$. Así, como supervisor de efectos visuales, ha asistido a situaciones en las que los equipos de producción afrontaban esa inseguridad realizando multitud de copias de seguridad del material obtenido en rodaje, ante la inexistencia de estándares sobre los que basar un esquema razonado de almacenamiento.

Actualmente, uno de los ejemplos de tecnologías más robustas es el flujo de trabajo comercializado por Red One, que ha desarrollado un software específico para la gestión de los archivos digitales generados por sus cámaras (RedcineX-Pro). Para el montador Raúl de Torres este software es la clave para facilitar la estabilidad del sistema en todo el pipeline.

“... tengo más que constatado que hacen las cosas pensando en que luego el material rodado hay que montarlo y postproducirlo. Esto, que parece de cajón, no siempre pasa, ya que muchas veces somos víctimas de las alianzas y enfrentamientos entre marcas". (Romano, García y De Torres, 2012: 35).

Sin embargo, la apariencia de solidez del flujo de trabajo ofrecido por Red One es al mismo tiempo el reflejo de un escenario tecnológico sobresaturado de información que describe la compatibilidad entre una determinada cámara, grabando con una determinada configuración y usando un software de composición específico para una versión concreta ${ }^{14}$. Lejos de constituir un sistema sólido, este exceso de formatos y

${ }^{12}$ Finance y Zwerman (2010: 281) aluden a esta debilidad comparación con los sistemas analógicos de filmación, donde la norma de $\operatorname{los} 35 \mathrm{~mm}$ garantizaba la compatibilidad en los equipos implicados en el proceso.

13 Sony está empezando a ofertar bancos de imágenes a las productoras para almacenar sus brutos. Pero el problema radica en la inseguridad acerca del formato de las imágenes, que puede resultar incompatible dentro de pocos años.

${ }^{14}$ El supervisor de DI Igor Ridamovic realizó un test del comportamiento de archivos RAW grabados con una Red One que posteriormente serían gestionados en estaciones de trabajo Avid Media Composer o DS v. 10. Si bien la utilización de la Red One introducía un ahorro en los costes en el proceso de grabación, complicaba la postproducción en la medida en que requería de una gran capacidad de procesamiento. Además, el proceso implicó la utilización de software adicional para la gestión en paralelo de la metadata ya que la versión del Media composer no era capaz de leer los códigos de tiempo de los archivos quicktime utilizados para la realización de la edición off-line (Ridnanovic, 2008). Este tipo de contextos productivos siguen produciéndose, obligando a la implementación de test constantes en los planes de producción. 
condicionantes es, en definitiva, una evidencia de la falta de robustez de la tecnología digital en este caso. Al mismo tiempo, la aparente flexibilidad que presentan los sistemas digitales permiten multitud de posibilidades para resolver cada una de las fases que integran el flujo de trabajo. Por otro lado, ello puede provocar la implementación de herramientas que comprometan la compatibilidad de todo el sistema.

Un buen ejemplo en este sentido lo encontramos en la generalización del uso de archivos RAW en grabación. En la práctica, la introducción de formatos RAW está demostrando la fragilidad del entramado tecnológico digital, introduciendo factores que vendrían a alterar la fiabilidad de complejos sistemas digitales de postproducción que habían demostrado su solidez con anterioridad a la introducción de este tipo de formatos.

"Crea problemas porque no existen experiencias de este tipo sobre todo a nivel de comunicación. No es que diga que esta RAW es peor o no da el resultado esperado. Es más a nivel de compatibilidad con los equipos de postproducción; composición o corrección de color (...) sólo pido que no introduzcan problemas que ya estaban resueltos y que ahora tienes que volver a empezar". (J.M. Aragonés, entrevista personal, 21 de marzo de 2012).

En el ámbito académico español existe un proyecto que ha dedicado sus esfuerzos a mejorar las especificaciones técnicas de las rutinas productivas basadas en tecnología digital. El IMP (Intelligent Metadata-driven Processing and distribution of audiovisual media, proyecto perteneciente al "Seventh Framework Programme" que comenzó su actividad en el 2007), está financiado con fondos europeos y liderado por la Universitat Pompeu Fabra. El proyecto nació a principios del 2009 con el objetivo de crear un repositorio digital de imágenes que asegurase una gestión optimizada del tráfico de información referida a la configuración de la imagen digital basándose en el uso de metadata (IMP Project, 2011).

Con el uso de metadata (grupos de información destinados a describir o complementar un núcleo de información mayor o más pesado), el formato del material original permanece inalterado, siendo necesaria su manipulación sólo al final del proceso, lo que minimiza la necesidad de adaptar el formato de la imagen a cada fase de la producción. Es una tendencia generalizada la utilización de metadata que, creada en el momento del registro del material, es reutilizada en cada una de las etapas del flujo de trabajo. El IMP, sin embargo, propone otra filosofía en la que la metadata se crea para adaptar las especificaciones del material a las necesidades del usuario en cada momento de la producción. Para Bernard Aragonés, supervisor de efectos visuales e integrante del proyecto IMP: "It's not important only to manage your media but it is esencial to able to manage and control your metadata" (IMP Making of..., 2011). Las tecnologías y herramientas desarrolladas por el IMP se basan en la creación de un servidor digital al que se puede acceder en distintas fases de la producción cinematográfica. En el informe final de evaluación del proyecto publicado a mediados del 2011, se explicitan los buenos resultados obtenidos en la gestión del material audiovisual basado en el uso de metadatos compatibles en el flujo de trabajo "Edición-efectos digitales-corrección de color" (IMP project, 2011). 


\section{Conclusiones}

En el mercado existen herramientas en forma de software para la optimización de los procesos en la fase de postproducción, pero a día de hoy la industria y no sólo en lo que se refiere a esta etapa final de la creación cinematográfica, sigue necesitando del establecimiento de estándares y sistemas capaces de gestionar una enorme cantidad de datos con seguridad, solidez y eficiencia. La ausencia de sistemas compartidos por las distintas fases de producción cinematográfica estaría lastrando un aumento en el potencial industrial del cine a nivel global y en España.

La rapidez en la evolución de las herramientas digitales podría explicar el desorden tecnológico, pero debemos ser críticos con su implementación en la medida en que pueden limitar el potencial mismo de la llamada digitalización. Como resultado de las guerras comerciales y la falta de acuerdos entre fabricantes de hardware y software, no sólo el consumidor se enfrenta a un escenario complejo e inestable. Los mismos profesionales estarían sufriendo las consecuencias de la ausencia de estándares digitales, repercutiendo en su competitividad y lastrando su potencial industrial.

Creemos que el uso de formatos sin comprimir gestionados a través de sistemas basados en el uso de metada constituye una línea de trabajo fértil para el establecimiento de estándares digitales. De esta forma sería posible fijar un criterio desde el inicio del proceso de creación, racionalizando el uso de espacio de almacenamiento, permitiendo mantener niveles altos de calidad y simplificando a priori el establecimiento de sistemas de compatibilidad entre las partes implicadas.

La digitalización de la producción cinematográfica está provocando nuevos retos a nivel industrial que afrontados desde posturas innovadoras, constituyen una oportunidad para la evolución del sector cinematográfico español. Un cambio de actitud que nos permita pasar de espectadores de una revolución tecnológica, al desarrollo de líneas de investigación mediante una colaboración Universidad-Empresa más productiva, basada en objetivos concretos.

\section{Referencias bibliográficas}

BENNETT, Bruce, FURSTENAU, Marc y MACKENZIE, Adrian (2008). "Format" en BENNETT, Bruce, FURSTENAU, Marc y MACKENZIE, Adrian: Cinema and technology cultures, theories, practices. New York, Palgrave MacMillan, pp. 19-21.

BRINKMAN, Ron (2008): The art and science of digital compositing ( $2^{\mathrm{a}}$ ed.). EEUU, Elsevier.

CASE, Dominique (2003): Nuevas tecnologías aplicadas a la postproducción. Andoaín, Escuela de Cine y Video

CILLER, Carmen. (2009): "La producción en la postproducción. El caso de Alatriste". En MARZAL, Javier, y GÓMEZ, Francisco Javier : El productor y la producción en la industria cinematográfica. Madrid, Editorial Complutense, pp. 411-420.

"E1 25 por ciento de las salas en España están digitalizadas". Cineinforme n ${ }^{\circ}$ 859. Madrid, Grupo Exportfilm, pp. 32-34.

FERNÁNDEZ CASADO, José Luis y NOHALES ESCRIBANO, Tirso (2000): Postproducción digital, cine y video no lineal ( $1^{\mathrm{a}}$ ed.). Andoaín, Escuela de Cine y Vídeo. 
FINANCE, Charles \& ZWERMAN, Susan (2010): The visual effects producer. Burlington, Elsevier.

"IMP Project. (2011): "Intelligent Metadata Media: D63.1 Final Evaluation Report" en IMP Project . http://www.imp-project.eu/?p=483. [Fecha de consulta: 10 de noviembre de 2011].

"IMP Making of..." [Video] (2011) en IMP Project. http://www.impproject.eu/?p=486. [Fecha de consulta: 20 de abril de 2011].

Instituto de la Cinematografía y de las Artes Audiovisuales. (2011): "Boletín informativo: películas, recaudación, espectadores, datos de 2010" en Boletín ICAA.http://www.mcu.es/cine/docs/MC/BIC/2010/Boletin_2010.pdf. [Fecha de consulta: 12 de enero de 2012].

JAMES, Jack (2006): Digital intermediates for film and video. Burlington, Elsevier.

"La producción de largometrajes con medios digitales en España se estabilizó en 2010". Cineinforme $n^{\circ}$ 859. Madrid, Grupo Exportfilm, pp. 14-20.

LARUMBE, Nacho (2012): "Naturalidad con matices". Cameraman n57. Madrid, Pablo del Río, pp. 26-32.

NOVY, Dan (2010): "Virtual Studio Technology" en OKUN, Jeffrey \& ZWERMAN, Susan: The VES handbook of visual effects. Burlington, Elsevier, pp. 783-784.

ROMANO, Peris, GARCÍA, Antonio y De Torres, Raúl (2012): "En plan romántico. Guerrilla en 5K". Cameraman n59. Madrid, Pablo del Río, pp. 32-36".

RIDNANOVIC, Igor (2008):“Avid and Red Digital Cinema" en Millimeter http://digitalcontentproducer.com/issue_20081101_01/. [Fecha de consulta: 11 de abril de 2011]

SWARTZ, Charles (2005): Understanding digital cinema. Burlington, Elsevier.

SQUIRES, Scott (2010): "Resolution and image format considerations" en OKUN, Jeffrey \& ZWERMAN, Susan: The VES handbook of visual effects. Burlington, Elsevier.

SPELTHANN, Volker \& HAUNSCHILD, Axel (2011): "Organizational Creativity in Heterarchies: The Case of VFX production". Creativity and Innovation Management (10 vol. 2). New Jersey, Blackwell Publishing, pp. 100-107.

WRIGHT, Steve (2008): Compositing visual effects: Essentials for the aspiring artist. Burlington, Focal Press.

\section{$\overline{\text { Oswaldo GARCÍA CRESPO }}$}

oswaldogarciacrespo@gmail.com

Realizador audiovisual y profesor asociado en el Departamento de comunicación audiovisual e Publicidade de la Universidade de Vigo. 